\title{
La migration des jeunes d'une région à l'autre au Québec. Sept questions d'intérêt pour les municipalités.
}

\author{
Lucie Fréchette et Yao Assogba ${ }^{1}$ \\ Université du Québec en Outaouais
}

Le phénomène du déplacement des jeunes d'une localité à l'autre ou d'une région à l'autre n'est pas récent. À différentes époques, il a préoccupé les gouvernements locaux et régionaux. L'histoire des régions du Québec a été jalonnée de mouvements migratoires $^{2}$. Ceux-ci sont aujourd'hui associés non pas au développement de territoires, mais à la décroissance de la population totale de certaines régions et la diminution du poids démographique des jeunes. La mobilité géographique concerne les régions qui voient leurs jeunes quitter vers de grands centres urbains, situation importante au point où les régions rura-

Trois grandes raisons sont invoquées comme motifs de départ : poursuivre des études, vivre sa vie de façon autonome et trouver du travail. tuel retour, etc. Les travaux ont alors pris la forme d'entrevues auprès de 103 jeunes de diverses régions du Québec. Finalement, l'étude a pris de l'ampleur au moyen d'un sondage téléphonique en collaboration avec la maison Sondagem. Effectué entre l'automne 1998 et le printemps 1999, le sondage a rejoint 5518 jeunes de 16 à 34 ans. Ce total se découpe en deux échantillons. Un premier échantillon national comprenait 2322 personnes dans les 17 régions québécoises. S'est ajouté un suréchantillonnage de 3196 personnes en provenance de 10 des 17 régions. C'est à partir de l'ensemble des travaux du GRMJ et des résultats obtenus que nous traitons dans ce texte de la migration des jeunes en sept questions susceptibles d'intéresser les municipalités et les MRC.

\section{Pourquoi partir ?}

Même si les jeunes demeurent en général plus longtemps au domicile familial, un grand nombre le quitte avant d'avoir atteint la vingtaine. Selon les travaux du GRMJ, le premier départ des jeunes s'effectue en grande majorité à l'extérieur de leur région, seul un tiers des jeunes demeurent dans leur région administrative d'origine. Trois grandes raisons sont invoquées comme motifs de départ : poursuivre des études, vivre sa vie de façon autonome et trouver du travail. Quand la migration s'effectue à l'intérieur de sa région, un autre motif prend aussi de l'importance : accompagner un conjoint.

Les motifs évoqués précédemment semblent simples. Ils recouvrent toutefois des réalités différentes d'un jeune à l'autre ou d'une région à l'autre. Dans l'ensemble du Québec, la poursuite des études est le motif de départ du quart des jeunes se déplaçant à l'intérieur de 
leur région d'origine, tandis qu'il est celui de la moitié de ceux qui quittent la région d'origine. Si l'on s'en tient à la situation des jeunes ruraux, le portrait diffère : $54 \%$ quittent pour poursuivre des études, $19 \%$ pour trouver du travail et $17 \%$ pour accompagner un conjoint.

Les études du GRMJ auprès des jeunes ayant quitté leur région confirment que leur perception est encore fortement teintée de l'idée qu'il n'y a pas possibilité de répondre à leurs aspirations professionnelles dans leur milieu d'origine, comme Roy ${ }^{4}$ l'avait déjà indiqué à partir de travaux menés en Estrie et dans le Bas-SaintLaurent. En gros, un jeune sur deux est de cet avis. Les jeunes de la Gaspésie-Iles-de-la-Madeleine, du Bas-Saint-Laurent, de la Mauricie et de l'AbitibiTémiscamingue sont ceux chez qui l'idée est la plus répandue. Par contre, les jeunes qui se sont déplacés à l'intérieur de leur propre région ont une évaluation plus nuancée, quoique encore environ $40 \%$ des jeunes ne croient pas qu'ils puissent s'y accomplir de façon souhaitable.

Les données du GRMJ révèlent cependant que l'opinion des jeunes a évolué quant à leur évaluation des activités culturelles accessibles dans leur région. Environ $30 \%$ des jeunes en ont une perception néga- tive. Il en va de même de l'attachement à la région d'origine qui, dans nos études, est décelable chez de nombreux jeunes et qui se modifie graduellement au cours des trajectoires migratoires ${ }^{5}$.

\section{Les jeunes migrants songent-ils à revenir dans leur région?}

Une question du sondage national demandait aux jeunes s'ils reviendraient vivre dans leur localité d'origine si les circonstances s'y prêtaient. La question est aussi présente à l'esprit des membres des gouvernements locaux ou régionaux affectés par le départ des jeunes dans leurs territoires respectifs.

Les jeunes qui vivent dans leur région d'origine sont plus ouverts à un retour dans leur localité d'origine (70\%) que ceux qui ont quitté leur région, même si environ $59 \%$ d'entre eux n'y sont pas fermés. Les variations régionales sont suffisamment importantes pour que nous les résumions sous forme de tableaux pour les régions administratives Bas-Saint-Laurent (01), Saguenay-Lac-St-Jean (02), Québec (03), Mauricie (04), Outaouais (07), Abitibi-Témiscamingue (08), Côte-Nord (09), Gaspésie-Iles-de-la-Madeleine (11), Centre-du-Québec (17).

Tableau 1 - Reviendriez-vous vivre dans votre localité d'origine si les circonstances s'y prêtaient ? Taux de réponse des jeunes ayant migré dans une autre région

\begin{tabular}{lcccccccccc} 
Région & $\mathbf{0 1}$ & $\mathbf{0 2}$ & $\mathbf{0 3}$ & $\mathbf{0 4}$ & $\mathbf{0 7}$ & $\mathbf{0 8}$ & $\mathbf{0 9}$ & $\mathbf{1 1}$ & $\mathbf{1 2}$ & $\mathbf{1 7}$ \\
\hline Oui & $66,7 \%$ & $63,4 \%$ & $66,7 \%$ & $66,7 \%$ & $71,4 \%$ & $52,9 \%$ & $57,2 \%$ & $61,1 \%$ & $70 \%$ & $57,6 \%$ \\
Non & $33,3 \%$ & $31,7 \%$ & $33,3 \%$ & $33,3 \%$ & $23,8 \%$ & $47,1 \%$ & $35,7 \%$ & $27,8 \%$ & $30 \%$ & $39,4 \%$ \\
Peut-être & - & $4,9 \%$ & - & - & $4,8 \%$ & - & $7,1 \%$ & $11,1 \%$ & - & $3 \%$ \\
\hline
\end{tabular}

Tableau 2 - Reviendriez-vous vivre dans votre localité d'origine si les circonstances s'y prêtaient ? Taux de réponse des jeunes s'étant déplacés à l'intérieur de leur région d'origine

$\begin{array}{lcccccccccc}\text { Région } & \mathbf{0 1} & \mathbf{0 2} & \mathbf{0 3} & \mathbf{0 4} & \mathbf{0 7} & \mathbf{0 8} & \mathbf{0 9} & \mathbf{1 1} & \mathbf{1 2} & \mathbf{1 7} \\ \text { Oui } & 60 \% & 55,2 \% & 53,1 \% & 70,8 \% & 63,2 \% & 61,5 \% & 50 \% & 60 \% & 70,6 \% & 77,8 \% \\ \text { Non } & 33,3 \% & 37,9 \% & 43,8 \% & 25 \% & 31,6 \% & 38,5 \% & 50 \% & 40 \% & 29,4 \% & 16,7 \% \\ \text { Peut-être } & 6,7 \% & 6,9 \% & 3,1 \% & 4,2 \% & 5,3 \% & - & - & - & - & 5,6 \%\end{array}$

Source : GRMJ. Sondage sur la migration des jeunes, 1998-1999. 


\section{Quelles raisons justifient le retour des jeunes dans leur région?}

L'explication la plus souvent fournie pour justifier un éventuel retour en région est la possibilité d'y gagner sa vie. On invoque, comme second motif, celle de pouvoir vivre avec des gens que l'on aime, puis de posséder une maison à soi et élever ses enfants. Les mêmes raisons sont nommées par les migrants intrarégionaux en ce qui concerne la possibilité d'un retour à la localité d'origine ou ses environs. Il semble que ces motifs correspondent à la réalité vécue par ceux qui ont effectué un retour après avoir fait l'expérience de la migration.

Leblanc et al. ${ }^{6}$ ainsi que Leblanc et Girard ${ }^{7}$ et Côté et Potvin ${ }^{8}$ ont regardé cette question de plus près chez les jeunes de milieux ruraux. Selon les travaux du GRMJ, l'emploi est ce qui a ramené dans leur coin de pays plus de $60 \%$ des répondants de retour après un séjour hors de leur région (plus de $80 \%$ en Outaouais et en Gaspésie et plus de $70 \%$ en Abitibi-Témiscamingue et sur la Côte-Nord). La même raison est aussi celle qui justifie le plus souvent le retour dans la localité d'origine ceux qui s'étaient déplacés à l'intérieur de leur région.

\section{L'explication la plus souvent fournie pour justifier un éventuel retour en région est la possibilité d'y gagner sa vie. On invoque, comme second motif, celle de pouvoir vivre avec des gens que l'on aime, puis de posséder une maison à soi et élever ses enfants.}

Avoir une maison à soi est aussi un facteur invoqué par plus de la moitié des jeunes ruraux de retour (plus de $50 \%$ en Outaouais, en Gaspésie et au SaguenayLac-Saint-Jean). Suit de près le rapprochement des parents et des amis (plus de $50 \%$ dans le Bas SaintLaurent, au Saguenay-Lac-Saint-Jean et en Gaspésie). Viennent ensuite le fait de fonder une famille, suivre un conjoint et démarrer une petite entreprise. Les raisons voient aussi leur poids se modifier selon l'âge des répondants. Par exemple, fonder une famille et posséder une maison sont des raisons dont la fréquence augmente avec l'âge des répondants. Finalement de $15 \%$ à $20 \%$ des jeunes reviennent pour dé- marrer une entreprise ou se lancer en affaires, pourcentage non négligeable.

L'ensemble de ces motifs de retour donne matière à réfléchir aux régions qui veulent entretenir l'intérêt des jeunes pour la vie en région. Il n'est pas surprenant que l'emploi soit le facteur principal de rétention des jeunes ou de retour des jeunes. Les autres facteurs ne sont pas pour autant sans intérêt. Les mandats de municipalités sont assez larges pour que celles-ci fassent le choix d'investir dans certains secteurs de développement social qui influencent la qualité de vie au sens des attentes des jeunes adultes et des jeunes familles. On ne peut cependant traiter isolément ces facteurs. Ainsi, des facteurs comme la représentation de sa région et de son avenir, l'attachement plus ou moins fort à sa localité ou à sa région ainsi que le sens de l'appartenance sont eux aussi influents.

\section{Comment perçoit-on la région d'origine et les autres milieux où l'on a transité ?}

\section{Le point de vue global}

On ne peut dissocier l'intention de retour de l'évaluation que font les jeunes de leur milieu d'origine, ni de la façon dont ils évaluent les autres milieux qu'ils ont habités par la suite. Le sondage du GRMJ indique que les jeunes identifient des contrastes significatifs entre les grandes villes et les milieux ruraux. Ils mentionnent que les services sont davantage à proximité des gens dans les grandes villes et que les activités culturelles y sont plus variées et accessibles. Ils reconnaissent, par contre, que la proximité de la nature est nettement plus forte en région, tout comme il est beaucoup plus facile d'y mener une vie paisible. Ces perceptions sont aussi généralement répandues dans la population en général au sujet de la qualité de vie «à la ville et à la campagne ».

Les entrevues auprès des migrants québécois ont aussi démontré qu'ils jugent comme des caractéristiques du milieu rural ou des petites villes la présence des espaces verts, la proximité de la nature, les opportunités de sports et d'activités en plein air et l'aspect sécuritaire des lieux. Ces perceptions rejoignent l'évaluation que font les jeunes ruraux canadiens de la vie en milieu rural ${ }^{9}$. On retrouve, dans un document de recherche du Partenariat rural canadien, une évaluation des milieux ruraux qui les présente comme des 
collectivités empreintes de sécurité, propices pour élever une famille et fournissant un environnement sain.

\section{Le point de vue sur les lacunes}

L'enquête auprès des jeunes est allée au-delà de ces généralités pour sonder les lacunes des régions d'origine du point de vue des jeunes. Les deux opinions les plus fréquentes dans l'ensemble du Québec sont que les régions sont marquées par la lenteur des décideurs et que la situation économique qui prévaut en région est difficile. Dans les régions rurales, plus de $40 \%$ des jeunes émettent ensuite des opinions traduisant une évaluation négative de la situation de l'emploi en région. On mentionne le manque d'emplois pour soi ou son conjoint et le fait qu'il est impossible d'entrevoir de l'avancement. En général, plus la scolarité augmente, plus l'on croit que le milieu d'origine est limité quant à la possibilité d'avancement. LeBlanc ${ }^{10}$ mentionne aussi que plus les jeunes se sont éloignés de leur milieu d'origine, plus ils sont négatifs au sujet de l'emploi à cet endroit. Finalement, selon les données du sondage téléphonique, les opinions sont diversifiées quant à la présence suffisante de services dans les régions. Partout les services scolaires de base sont jugés accessibles; par contre, l'évaluation que l'on fait des services de santé et des services de loisirs varie selon les régions et selon les caractéristiques des répondants.

La perception des lacunes du milieu d'origine transpire aussi de façon manifeste des entrevues menées auprès des jeunes. Comme dans le sondage téléphonique, la plupart des jeunes ont évoqué la rareté des emplois ou les faibles perspectives d'emploi. En entrevue, plusieurs jeunes signalent également l'insuffisance des lieux de rencontre et d'activités de loisirs conçus pour les jeunes. Ils qualifient leur milieu d'origine, surtout en régions essentiellement rurales, de peu animé, monotone et manquant de diversité dans l'offre d'activités. Par ailleurs, les jeunes soulèvent la rareté et l'éloignement des services et des centres commerciaux ainsi que l'absence de transport en commun. Finalement, des jeunes de milieux peu populeux déplorent la difficulté de conserver une vie privée dans un milieu où tout le monde se connaît. Enfin, sont également peu appréciés l'intolérance et le manque d'ouverture d'esprit de la population vieillissante.

Encore une fois, ces résultats rejoignent des données des études du Partenariat rural canadien qui rapportent que les jeunes ont, des régions rurales, l'image de milieux où il est difficile de bien gagner sa vie ou de poursuivre des études et d'envisager des perspectives de carrière intéressante tant en termes économiques que sociaux. Le thème est aussi récurrent dans l'analyse de la migration des jeunes de milieu rural québécois. Les jeunes les plus scolarisés, chez les étudiants et les chercheurs d'emploi, estiment que les possibilités d'avancement sont fort limitées dans leur milieu d'origine. Ces travaux indiquent aussi les préoccupations des jeunes quant à la présence plus modeste de services scolaires ou socio-sanitaires et quant à la rareté des services associés au loisir et à la culture.

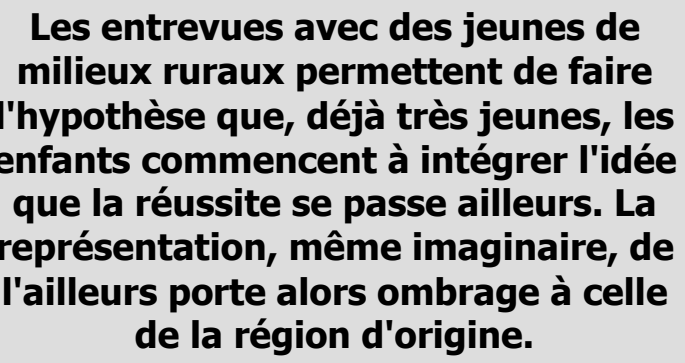

\section{Réussir, c'est partir... Une représentation à contrer}

Les entrevues avec des jeunes de milieux ruraux permettent de faire l'hypothèse que, déjà très jeunes, les enfants commencent à intégrer l'idée que la réussite se passe ailleurs. La représentation, même imaginaire, de l'ailleurs porte alors ombrage à celle de la région d'origine. Dès l'école primaire, il arrive que le discours prépare le départ des jeunes de la région. Il en va de même dans le discours de familles qui préparent tôt leurs enfants à partir pour réussir leur vie. Un extrait d'entrevue donne à réfléchir : « À X, on s'est toujours fait dire que tu n'allais nulle part si tu restais à $X$. C'était négatif. Surtout qu'on voyait qu'il n'y avait pas grand-chose pour les jeunes. C'était pas un bon message qu'on avait pour les jeunes ». LeBlanc ${ }^{11}$ souligne le même phénomène autour de l'emploi. Le discours le plus répandu veut qu'il soit difficile de trouver du travail en région. Or, l'expérience de certains jeunes plaide plutôt pour nuancer ce discours. Plusieurs jeunes font l'expérience du soutien à la recherche d'emploi lors de leur retour dans la région 
ou la localité d'origine. Les réseaux de connaissances y sont pour quelque chose.

\section{Que vivent les jeunes migrants lors de l'arrivée dans un nouveau milieu ?}

Les départs sont souvent dirigés vers Montréal ou Québec ou de grandes ou moyennes agglomérations comme Gatineau, Sherbrooke, Rimouski ou TroisRivières, villes centrales chacune dans leur région. La migration induit fréquemment l'expérience de la ville. Il s'agit d'une expérience de la différence et de l'urbanité, quelle que soit l'échelle où elle est expérimentée. Il ne faut donc pas se surprendre si le discours des jeunes s'installant dans une ville moyenne est à l'occasion le même que celui des jeunes qui découvrent Montréal. L'arrivée des jeunes dans un nouveau milieu exige une adaptation progressive de leurs représentations de l'univers urbain, de leurs attitudes et de leurs comportements aux nouvelles situations dans lesquelles ils se trouvent. Pensons à l'accommodation aux conditions de logement, à la vie conjugale ou familiale, à l'adaptation au contexte d'études, de la profession et du milieu de travail, à la recherche de loisirs, à la transaction avec des réseaux de sociabilité anciens ou nouvellement constitués, etc.

\section{Certains milieux sont conscients de la transition vécue par les gens qui y arrivent.}

\section{Composer avec l'éloignement}

Une des caractéristiques fondamentales de la migration est l'éloignement ou la séparation, au moins physique, du migrant des groupes d'appartenance de son milieu d'origine. L'éloignement est d'abord vécu difficilement par la plupart des jeunes migrants dans les centres urbains. Certains s'expriment en termes de nostalgie, d'ennui de la famille ou des amis, d'autres disent éprouver des sentiments de solitude et d'isolement. Un extrait d'entrevue traduit bien la prégnance de ces sentiments : "Je me sentais tout seul. J'ai fêté mes 20 ans complètement tout seul. [...] Je suis sorti dehors avec ma bière et je me suis mis à brailler, mais brailler... »

Lors des premiers mois ou de la première année, on cherche à maintenir les contacts avec le milieu d'ori- gine surtout par des communications téléphoniques avec la famille et le retour fréquent au lieu d'origine pour revoir la famille et/ou les amis. Les relations avec la famille se réorganisent progressivement sur une base différente. Du côté des relations amicales, une majorité des jeunes migrants conservent des liens amicaux dans le lieu d'origine, mais on constate que ces relations diminuent en nombre et s'estompent progressivement. Les contacts sont moins fréquents ou encore, le groupe d'amis se dissout, plusieurs d'entre eux ayant aussi quitté la région.

L'émergence des nouveaux groupes d'amis dans le milieu d'accueil représente alors une étape significative dans l'intégration ailleurs des jeunes migrants. Passer d'un réseau de sociabilité à un autre s'accomplit comme si le jeune migrant commençait tranquillement à couper le cordon invisible qui le liait encore à son patelin ${ }^{12}$.

\section{Composer avec la nouveauté}

Même si les jeunes s'attendaient à trouver de la nouveauté et des différences en ville, plusieurs d'entre eux sont déstabilisés lors de leur arrivée dans les grands centres urbains, spécialement à Montréal. La différence est plus grande que ce à quoi ils s'attendaient. Il ne s'agit pas que d'un cadre de vie différent, mais aussi d'une confrontation à d'autres modes de vie. Finalement, dans de grands centres, des phénomènes urbains comme la diversité culturelle, l'itinérance, la mendicité, la violence et la criminalité (dont on peut être témoin ou ce qu'en disent les médias) créent un choc et un sentiment d'insécurité ralentissant la (re) sociabilité des jeunes.

S'adapter à la nouveauté, c'est s'adapter à un nouveau cadre scolaire chez ceux qui entrent au cégep ou à l'université. C'est s'adapter à un environnement de travail et à ses exigences pour ceux qui migrent pour occuper un premier emploi. On s'inscrit dans de nouvelles normes de comportement. On côtoie des gens différents. On assume de nouvelles responsabilités. Certains milieux sont conscients de la transition vécue par les gens qui y arrivent. Ils mettent en œuvre des dispositifs d'accueil et parfois même, instaurent des systèmes d'accompagnement ou de mentorat qui facilitent l'insertion dans le nouveau milieu.

Aborder la nouveauté s'expérimente à travers l'apprivoisement d'espaces à diverses échelles. Plus les mi- 
grants sont jeunes, plus ils se trouvent davantage à l'aise dans des espaces éclatés plutôt qu'à l'intérieur de la dynamique de tel ou tel quartier. Ils fréquentent puis adoptent plusieurs quartiers selon qu'ils les habitent, y étudient, y travaillent ou y consomment des activités culturelles ou de loisirs. Parmi les endroits évoqués par les migrants comme espaces permettant d'apprivoiser la ville, de canaliser le sentiment d'étrangeté de la ville, de l'apprivoiser et de s'y resocialiser, trois micro-territoires s'imposent: le lieu de travail ou d'étude et les lieux de loisir au sens large du terme, autant le loisir-activité que le loisirsocialisation ${ }^{13}$. Par contre, les jeunes familles ont un peu plus tendance à rechercher une vie de quartiers dotés de logements adaptés aux familles, de services de garde d'enfants, de services commerciaux comme des épiceries, etc.

\section{Comment les jeunes s'intègrent-ils dans de nouveaux milieux?}

Dans le cas de la migration du jeune Québécois de région vers la ville, l'intégration consisterait en l'adaptation progressive de leurs représentations de l'univers urbain, de leurs attitudes et comportements aux nouvelles situations dans lesquelles il se trouve. Il peut s'agir de l'accommodation aux conditions de logement, à la vie conjugale ou familiale, l'adaptation au contexte d'études, au système d'enseignement, de la profession et du milieu de travail, de loisirs, aux réseaux de sociabilité anciens ou nouvellement constitués, etc. L'intégration du jeune migrant devient une adaptation à un nouveau milieu qui consiste en une relative acceptation des valeurs, des normes, bref de la culture urbaine.

\section{Explorer un environnement et ses services}

L'intégration suppose un rapport actif à l'environnement de proximité, sa fréquentation et une appréciation de la différenciation des pratiques qui y sont déployées. Le rapport positif à l'environnement de proximité crée une structuration globale de l'espace, sans avoir recours à des repères précis pour le représenter et à une acception élargie des environs, au-delà du voisinage immédiat, du quartier ou du village. Le rapport négatif, par contre, s'accompagne de l'absence de pratiques sur le lieu ou de pratiques minimales comme le dépannage alimentaire. Il s'ensuit parfois un certain rejet associé à un sentiment de méconnais- sance du territoire et une représentation mentale morcelée composée de repères précis mais peu nombreux.

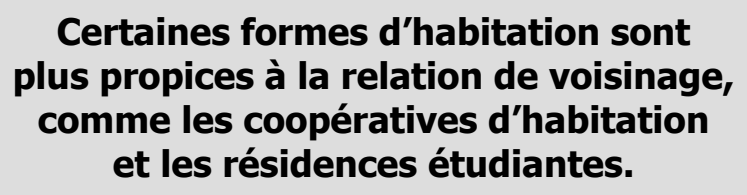

L'exploration plus ou moins systématique de l'environnement urbain est question de rythme. Les jeunes qui explorent l'espace urbain de manière systématique en retirent un bénéfice personnel et se disent à l'aise dans la ville, parfois même après seulement quelques semaines. Un des bénéfices secondaires de la fréquentation des infrastructures et des activités offertes par la ville est la diminution des peurs et l'avènement progressif d'un intérêt grandissant pour l'environnement urbain. Pour d'autres, la tâche demeure ardue, et c'est avec appréhension qu'ils se risquent hors du voisinage ou hors du trajet devenu familier pour se rendre au travail ou au lieu d'étude. Le voisinage immédiat est un espace qui, en ville, se révèle pertinent par défaut. On parle à son voisin de palier, d'abord par manque d'interlocuteurs signifiants autour de soi. Nos recherches nous ont tout de même laissé voir que certaines formes d'habitation sont plus propices à la relation de voisinage, comme les coopératives d'habitation et les résidences étudiantes. Dans ces environnements, la proximité de voisinage devient un facteur d'apprivoisement de la différence. Sous l'effet de la proximité se diluent la crainte de l'inconnu et la connotation négative qui l'accompagne.

Un autre territoire plus psychique est significatif du processus d'urbanisation rapporté par les jeunes migrants : celui du temps libre canalisé dans le loisir. Nous constatons aussi que la relation au loisir et à la culture du migré évolue dans le temps et reflète des changements de mentalité et de culture révélateurs d'un rapport renouvelé à l'urbanité. En début d'itinéraire, le loisir agit comme une activité compensatoire. Compensatoire pour les carences expérimentées dans le milieu d'origine où l'offre d'activités de loisir ou culturelles est jugée pauvre. Par contraste, la ville offre une diversité d'activités qui fascinent les nouveaux arrivants, peu importe la taille de la ville. « $\grave{A}$ Rouyn-Noranda, c'est une grande ville... Tu as plein de loisirs ici », souligne un jeune, tandis que d'autres déplorent les carences de villes de taille moyenne, 
comme Rouyn par comparaison à Montréal. Le regret récurrent chez les migrés est que Montréal, la grande ville, n'offre pas l'accessibilité à la nature. Le loisir est aussi une activité compensatoire pour la faiblesse initiale du réseau social dans le nouveau milieu. Les lieux de loisir sont rapidement investis pour se changer les idées et rencontrer des gens.

Par le loisir, les jeunes explorent la nouveauté de la ville et s'insèrent dans des lieux où se reconnaît et s'exprime la jeunesse. L'espace est alors découpé en lieux formels et informels médiatisés par le temps libre perçu. Ce dernier crée des perceptions de liberté, de choix de spontanéité, de densité émotive et sociale et de qualité de vie, qu'on aborde ce temps libre en général ou dans ses dimensions spécifiques comme le temps, la nature, le sport, la culture, les sorties, etc.

Finalement, des lieux où l'on peut exercer du bénévolat ou devenir participant d'un projet collectif, qu'il s'agisse des loisirs communautaires ou d'organisations du milieu associatif, deviennent des adjuvants à l'intégration. Ils apparaissent souvent comme des oasis dans un milieu où l'on se sent seul. La culture et les loisirs sont d'ailleurs présents à ce titre dans l'analyse sociale de la ville présentée comme un système complexe qui tente de rencontrer les besoins des gens qui y vivent ${ }^{14}$. La garderie, la coopérative d'habitation ou des associations communautaires sont rapportées comme des endroits où des migrants ont connu d'autres personnes et où ils ont commencé à se sentir partie prenante d'un milieu quelconque. Pour certains, on peut même prédire qu'il s'agit là d'expériences qui précèdent ou amorcent l'intégration dans un territoire qui tiendra lieu de communauté locale.

\section{À propos d'éléments participant de la reconstruction d'un réseau social}

Un sous-groupe du GRMJ a analysé les trajectoires d'intégration des jeunes dans les milieux d'accueil ${ }^{15}$, la migration étant aussi l'affaire des municipalités ou des régions d'accueil.

La dynamique de la reconstruction des réseaux sociaux semble être reliée dans une certaine mesure à la perception que créent chez les migrants les populations urbaines d'accueil. En termes plus explicites, cette reconstruction paraît plus laborieuse lorsque le migrant perçoit les gens du lieu d'accueil comme étant peu accueillants, fermés, trop différents, etc.
Les difficultés ou le défaut de reconstruire dans l'immédiat le lien social avec les résidents semblent spontanément ou naturellement se pallier par la création de nouveaux réseaux sociaux autour des autres migrants que l'on rencontre à diverses occasions, par exemple à la résidence étudiante, dans un lieu de loisir ou de sport, dans le milieu de travail, etc. Tout se passe comme si les gens venus d'ailleurs tendent à se retrouver et à se regrouper, dans la mesure où la plupart des jeunes migrants dans les centres urbains partagent individuellement et collectivement le sentiment de rejet et d'incompréhension de la part des résidents locaux. C'est dans ce contexte que l'effet de rencontre se produit généralement entre les migrants issus de la même région. C'est sans doute ce qui explique en partie la vitalité des associations de migrants dans divers centres du Québec comme Les Bleuets du Saguenay regroupés dans l'Outaouais ou les Gaspésiens ou Abitibiens qui se sont donné des associations à Montréal.

L'effet de rencontre se produit également entre les migrants issus de localités ou de régions différentes. Un extrait d'entrevue rend bien l'idée : « Des gens de l'extérieur ici, il y en a beaucoup et eux aussi veulent connaître du monde parce qu'ils ne connaissent personne, même chose que moi... ». La reconstitution des liens sociaux autour d'un noyau de migrants semble faciliter l'adaptation d'un jeune de région qui a migré dans un centre urbain. On observe que la présence dans le lieu de chute d'un membre de la parenté, ou d'un groupe d'amis de la région d'origine du migrant, facilite encore davantage l'intégration de celui-ci.

La vie de couple permet également aux migrants d'avoir plus facilement accès à un réseau social dans le lieu de chute. Dans certains cas, l'un des conjoints vient rejoindre le premier déjà installé et souvent inséré dans un nouveau milieu de travail. Le fait de former un nouveau couple avec un ou une partenaire locale favorise aussi la mise en réseau.

La précarité d'emploi change cependant les choses. Les étudiants et les jeunes travailleurs qui connaissent une succession d'emplois précaires investiront peu d'énergie pour se révéler à d'autres au-delà de la connaissance éphémère. Ce qui n'est pas sans affecter le choix résidentiel, les conditions de vie et le réseau social, éléments associés à l'intégration.

Il ressort de l'analyse qui précède que l'intégration dans un milieu d'accueil n'est pas qu'une insertion 
utilitariste en un territoire géographique donné. Elle ne peut faire l'économie de la formation des réseaux sociaux, fondés sur des liens sociaux d'intensité variable, à l'intérieur de lieux significatifs de vie la sociale. Ce processus s'inscrit dans une dynamique complexe faite de logiques et de stratégies du migrant et d'effets $\mathrm{du}$ hasard, mais aussi d'une planification d'actions favorisant la mise en relation dans des institutions ou équipements qui accueillent de nouveaux arrivants d'autres régions du Québec.

L'intégration dans un milieu d'accueil n'est pas qu'une insertion utilitariste en un territoire géographique donné.

\section{La migration change-t-elle les jeunes? Questions d'identité et de sentiment d'appartenance}

L'ouverture du réseau social n'est pas qu'affaire de relations interpersonnelles ou de réseau de soutien. Les jeunes migrants s'ouvrent à la diversité. La nature de la relation subjective que les jeunes migrants entretiennent avec la collectivité du lieu d'origine puis avec celle du lieu d'accueil affecte son processus identitaire et, de façon plus générale, son sentiment d'appartenance. Autrement dit, pendant le processus d'intégration, s'opère un changement dans la définition de leur identité et de leurs appartenances, alors qu'ils entreprennent une lente sortie d'eux-mêmes et de la culture de leur milieu d'origine.

Malgré l'éloignement physique, la plupart des jeunes migrants affichent une identification à la région d'origine et déplorent l'ignorance de la population urbaine quand les gens de la ville ne connaissent pas ou ne sont pas en mesure de situer leur village ou région d'origine. Ils déplorent aussi les préjugés et de fausses images qui circulent au sujet de leur communauté d'origine. Les jeunes ressentent la dévalorisation du monde rural traduite par certains messages véhiculés au sein même des communautés régionales. Par exemple, nous avons fait référence auparavant dans le texte aux messages que la réussite se passait ailleurs que dans certaines régions d'origine.

L'expérience des jeunes migrants montre qu'un certain degré de détachement s'opère graduellement et qu'un sentiment d'appartenance se développe à l'é- gard du milieu d'accueil. Reste que la région d'origine occupe encore une place importante aux yeux de la plupart des jeunes. La communauté de départ est associée à l'enfance, à la vie familiale et à ses souvenirs. Elle représente encore le lieu des rencontres familiales ou celui du réconfort. Les jeunes y restent attachés. Avec le temps, elle représente cependant davantage le lieu de résidence des parents que leur véritable « chez eux ». À cet égard, des jeunes disent avoir le sentiment d'être " en visite » lorsqu'ils retournent dans leur milieu d'origine.

Les jeunes migrants qui portent un regard plus négatif sur leur milieu d'origine estiment que la vie privée est envahie. Un milieu où toute la population encadre les manières de penser et d'agir limite les aspirations et l'affirmation de soi et est vécu comme nuisible à l'affirmation de sa véritable identité. Dans d'autres cas, l'absence de lieux où l'on peut pratiquer des activités avec son groupe générationnel pose problème. Poussé à l'extrême, ce constat peut inciter le jeune à partir. La famille et les groupes de corésidence ne parviennent plus à créer un environnement où le jeune peut s'identifier à d'autres membres de sa génération. L'imaginaire d'un ailleurs idéalisé vient faire contrepoids à cette image négative de son milieu d'origine. La grande ville apparaît sous ce rapport le lieu de prédilection pour assumer cette différence.

Le sentiment d'appartenance chez nos répondants s'est développé à mesure que les jeunes migrants se sont insérés professionnellement ou se sont construit de nouveaux réseaux sociaux. Les jeunes migrants sont soumis à des repères d'identification qui varient (réseaux sociaux, cultures, langues, modèles de valeurs et de comportements différents). Le rapport au territoire constitue donc un enjeu dans le maintien de soi et la définition de l'identité. Chez les jeunes que nous avons étudiés se manifeste, en effet, autour de fragments et dans une certaine fluidité, la nécessité de construire leur identité dans un rapport de continuité (rapport au lieu et à la culture d'origine) / discontinuité (lieu et culture d'accueil) ${ }^{16}$.

Quand, ils disent se reconnaître comme membres de leur nouvelle région d'accueil, on voit naître un sentiment d'appartenance renouvelé. On commence à s'insérer dans une identité collective qui se renouvelle, faite de ce qu'on a de nouveau en commun avec d'autres. 


\section{En guise de conclusion}

Que faire de ces connaissances sur la migration? Un premier réflexe serait de chercher les moyens de retenir les jeunes dans les régions. La migration des jeunes, légitimée le plus souvent par la poursuite des études et parfois par la recherche d'un emploi, est aussi une quête d'autonomie chez les jeunes et participe de leur passage à la vie adulte. Chercher à retenir les jeunes à tout prix serait un réflexe malhabile qui ne tiendrait compte ni des besoins d'ouverture et d'autonomisation et de développement des jeunes, ni de l'enrichissement des régions construit sur des assises de compétences accrues des jeunes générations. Miser sur des actions qui favoriseraient le retour des jeunes en région ou attireraient en région des jeunes et des jeunes familles d'ailleurs serait plus avisé.

Un membre du GRMJ, qui centre ses travaux sur la migration des jeunes ruraux, soumet à la réflexion des pouvoirs régionaux trois pistes d'intervention ${ }^{17}: 1$ ) encourager le retour des jeunes dans leur milieu d'origine par le recours à des programmes destinés aux jeunes migrants, tel le programme Place aux jeunes. Il suggère aussi des mesures favorisant l'accès à la propriété pour les jeunes ménages et les jeunes familles et le soutien au remboursement des dettes d'études; 2) garder le lien pendant la migration; il conseille à cet effet l'utilisation de l'Internet, le jumelage avec des employeurs de la région, etc.; 3) développer le sentiment d'appartenance aux régions. Il recommande d'y travailler dès l'école primaire ou à partir de programmes destinés aux adolescents.

L'action pour assurer une place aux jeunes et aux jeunes familles en région passe bien sûr par l'emploi, mais elle passe aussi par des conditions de vie attrayantes du point de vue des jeunes et des familles. Les politiques familiales municipales et les politiques de loisir, de culture et de services communautaires sont des canevas idéaux pour mettre en place de telles conditions. Les plans d'action en suivi à ces politiques auraient avantage à prendre en compte le phénomène de la migration des jeunes. Un chalet à la disposition des jeunes familles venant visiter les grands-parents en milieu rural, un ordinateur installé à la bibliothèque municipale ou au bureau de poste avec un branchement Internet pour le courriel, un taxi collectif pour favoriser les déplacements des étudiants vers la ville centre les fins de semaine, une fête annuelle des retrouvailles, voilà autant de moyens créatifs et peu coûteux de maintenir le lien avec la région d'origine.

\section{Notes et références}

1 Lucie Fréchette est professeure au Département de travail social et des sciences sociales de l'Université du Québec en Outaouais et coordonnatrice du Centre d'étude et de recherche en intervention sociale (CERIS). Yao Assogba, professeur au même département, est responsable du programme de Maîtrise en travail social. Ils sont tous deux membres du Groupe de recherche sur la migration des jeunes (GRMJ).

2 Perron, N. (1997). «Les migrations depuis le XIX ${ }^{\mathrm{e}}$ siècle au Québec », dans Madeleine Gauthier (dir.), Pourquoi partir? La migration des jeunes d'hier et d'aujourd'hui, Sainte-Foy, Éditions de l'IQRC/PUL, p. 23-48.

3 Dirigé par Madeleine Gauthier de l'INRS Urbanisation, culture et société-Québec, le groupe compte des chercheurs de cinq constituantes du réseau de l'Université du Québec (UQO en Outaouais, UQAT en Abitibi-Témiscamingue, UQAR à Rimouski, UQTR à Trois-Rivières, UQAC à Chicoutimi), de l'Université de Sherbrooke et de l'Université d'Ottawa et, pendant quelques années, de l'UQAM.

4 Roy, J. (1997). «La quête d'un espace sociétal», dans Madeleine Gauthier (dir.) La migration des jeunes d'hier et d'aujourd'hui, Sainte-Foy, Éditions de l'IQRC/PUL, p. 87-103.

5 Côté (1997) «Migrer : un choix ou une nécessité? Une enquête à l'échelle d'une région », dans Madeleine Gauthier (dir.) La migration des jeunes d'hier et d'aujourd'hui, Sainte-Foy, Éditions de l'IQRC/PUL L, p. 63-85; LeBlanc, P. (2002). «La migration des jeunes ruraux de l'Abitibi-Témiscamingue et leur avenir en région », Le Trotteur, vol. 5, n ${ }^{\circ}$ 5; Assogba, Y., L. Fréchette et D. Desmarais (2000). « Le mouvement migratoire des jeunes au Québec. La reconfiguration du réseau social, un repère pour étudier le processus d'intégration », Nouvelles pratiques sociales, vol. $13, \mathrm{n}^{\circ} 2$, p. 65-78.

6 LeBlanc, P., M. Gauthier et D.-H. Mercier (2002). «La migration des jeunes de milieu rural », Cahier de l'INRS Urbanisation, culture et société, Montréal, 123 p.

7 LeBlanc, P. et C. Girard (2001). «La dynamique migratoire des jeunes au Québec. Le croissant péri-nordique de l'Abitibi-Témiscamingue, du Saguenay-Lac-Saint-Jean et du Bas-Saint-Laurent », L'Action nationale, vol. XCI, $\mathrm{n}^{\circ} 5, \mathrm{p} .23-42$.

8 Côté, S. et D. Potvin (2001). « Les multiples visages de la migration des jeunes en Gaspésie et dans trois régions de 
l'Est », dans Danielle Lafontaine (dir.), Choix publics et prospective territoriale. Horizon 2005. La Gaspésie : futurs anticipés, Rimouski, UQAR-GRIDEQ, p. 43-60.

9 Malestat, R.A. and Associates (2002). « La migration des jeunes ruraux : explorer la réalité derrière les mythes », rapport de recherche présenté au Partenariat rural canadien, Gouvernement du Canada.

10

LeBlanc, P. (2002), op. cit.

11 LeBlanc (2000) «Les jeunes de milieu rural et leur rapport à la région », dans Mario Carrier et Serge Côté (dir). Gouvernance et territoires ruraux: éléments d'un débat sur la responsabilité du développement, Sainte-Foy, PUQ, p.65-82.

12 Assogba, Y., L. Fréchette, L. et D. Desmarais (2000), op. cit.

13 Paré, J.-L. (1997). « Le temps de loisir », dans Madeleine Gauthier (dir.), Les 15-19 ans. Quel présent? Vers quel avenir?, Sainte-Foy, IQRC-PUL, p. 65-87.
14 Reichert, H. et J.-D. Remond (1980). Analyse sociale de la ville, Paris, Masson.

15 Fréchette, L. D. Desmarais, Y. Assogba et J.-L. Paré, «L'intégration des jeunes à la ville. Une dynamique de repérage spatial et social », dans $\mathrm{M}$. Molgat et $\mathrm{P}$. Leblanc, Aux frontières de l'espace et du temps, Québec, PUL (à paraître 2004); Desmarais, D., Y. Assogba et L. Fréchette (2001). «L'intégration des jeunes adultes migrants en milieu urbain au Québec », dans Henri Dorvil et Robert Mayer (dir.), Problèmes sociaux. Tome II : Études de cas et interventions sociale, Sainte-Foy, PUQ, p. 103-128; Assogba, Y., L. Fréchette et D. Desmarais (2000), op. cit..

16 Girard, C, S. Garneau et L. Fréchette, « Déplacement, identité et sentiment d'appartenance », dans Molgat et Leblanc, Aux frontières de l'espace et du temps, Québec, PUL (à paraître en 2004).

17 LeBlanc, P. (2002), op. cit. 